\title{
Building Skills in Engineering: Hand and Power Tool Workshops for Confi- dence and Retention
}

\section{Dr. Pamela L Dickrell, University of Florida}

Dr. Pamela Dickrell is the Associate Director of the Institute for Excellence in Engineering Education, in the UF Herbert Wertheim College of Engineering. Her role as Associate Director of the Institute focuses on effective teaching methods and hands-on learning opportunities for undergraduate student engagement and retention. Dr. Dickrell received her B.S., M.S., and Ph.D. in Mechanical Engineering from the University of Florida, specializing in Tribology. 


\title{
Building Skills in Engineering: Hand \& Power Tool Workshops for Confidence \& Retention
}

\begin{abstract}
This work examines a pilot program open to all undergraduate students in the Herbert Wertheim College of Engineering at the University of Florida on the use of common hand and power tools through a series of 7 weekly hands-on workshops led by a female faculty member as an open opportunity to gain building skills.
\end{abstract}

The goals of the program include:

a) Creating a safe environment for engineering students to learn, build, fail \& redesign, without pressure of grades or deliverables;

b) Increasing individual student tool knowledge, basic making skills, and confidence, so when in group projects later they do not shy away from the design and building portion of team based engineering projects;

c) Improving student skills for increased participation in engineering societies, internships, hands-on outreach projects, and student build teams; and

d) Promoting a culture of making within all engineering students.

Two groups of students were included in this study to examine female-only versus co-ed environment; a 20-person group of female students (Building Women in Engineering), and a 20person group of co-ed students (Building Skills in Engineering). Students from 10 different engineering majors, and all years of undergraduate studies were represented. Both groups had the same female faculty member and female undergraduate peer mentor leading the hands-on tool workshops with them each week. Students were surveyed before, at the mid-point, and following the 7 weeks of workshops to examine the impact of participation on their selfconfidence and interest in the hands-on aspects of engineering studies. Both qualitative (opened ended questions) and quantitative (likert questions) about impressions are included in this work. Additionally, outlines of the tools, build space, and individual workshop projects are included for peer universities interested in developing their own hand \& power tool build groups.

\section{Student Demographics}

The Building Women in Engineering and Building Skills in Engineering groups were advertised via email to the general undergraduate engineering population, and spots were filled on a firstcome, first-served basis. Both sections were limited to 20 students, with the Building Women in Engineering section being limited to female-only participants, and the Building Skills in Engineering section being open to all students for a co-ed group. Two different groups, one female only and one co-ed were selected to ensure all student demographics had the ability to participate, as well as giving female students opportunity to work in a female-only environment to build confidence learning within a group of gender peers. Spots for the build groups in this pilot were filled on a first-come first-served basis, so ethnic and year of study demographics represent the first 40 students to sign up for the build groups, they were not selected for any specific ethnic or year of study analysis in this pilot. Out of the total 40 students, 31 were female, 8 were male, and 1 student chose 'other or prefer not to answer'. The one student who chose 'other' approached the professor prior to the build group registration for advice on registration. 
The student at the time of the build group registration was in gender transition, and was unsure of which section to register for. Given the student self-identified as a male at the time of registration, the student chose to register for the co-ed section. The student decided if they participate in future semesters and feel they then self-identified as female, they might choose the female only section in future semesters. This information is included because part of the goal of this work is creating a working environment welcoming to all students, and this student voiced their input on the gender environment relating specifically to their personal transition in the 'Gender Environment Feedback' portion near the end of this work.
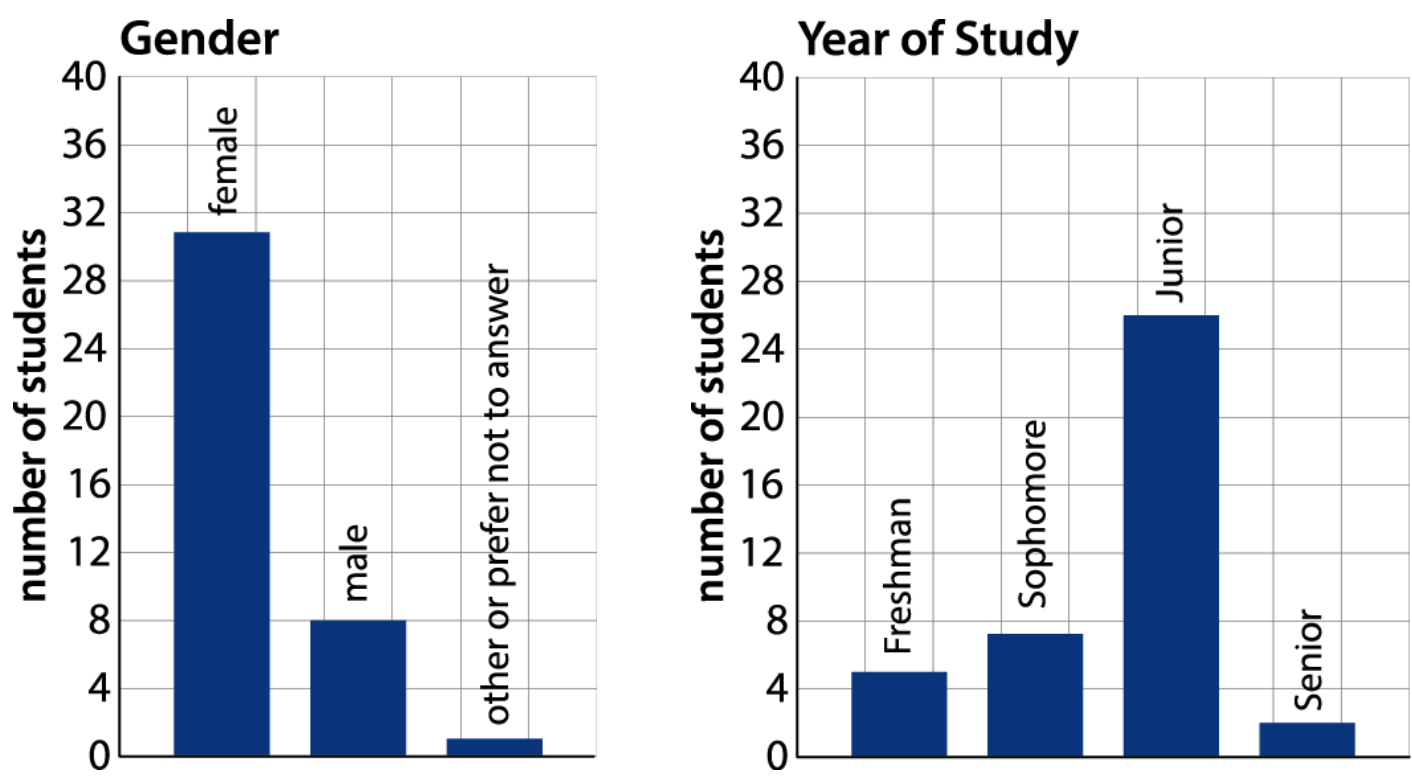

Combined student gender and year of study demographics from both build groups.

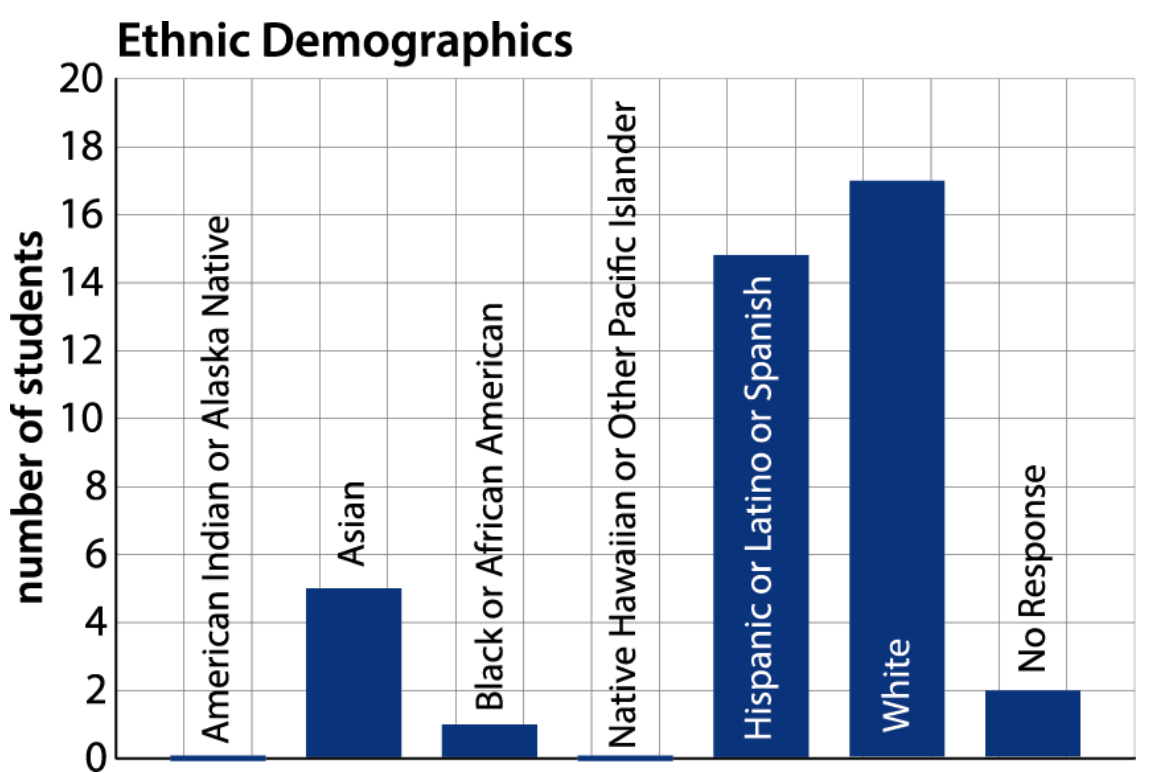

Combined student self-reported ethnic background demographics. 


\section{Active Learning Building Classroom}

The build groups were held in a designated build space classroom, which was a space marked for future renovation with a locking door, wall outlets, and a hard-surface floor, and little else. The student work spaces were set up in a modular format for future transition to a more permanent space or for easy room re-arrangement if needed for other learning applications. Five work stations were constructed, for seating groups of four students per table. Each 4 student station consisted of two mobile wooden-top student work tables (two students facing two students at each station) and a wooden-top rolling tool chest at the end of each four student station. The five work stations were distributed evenly around the perimeter of the room, with the tool chests facing the inner open space in the room. Wall mounted magnetic marker boards and pin-cushion boards were added for posting project-specific information and for student collaboration.

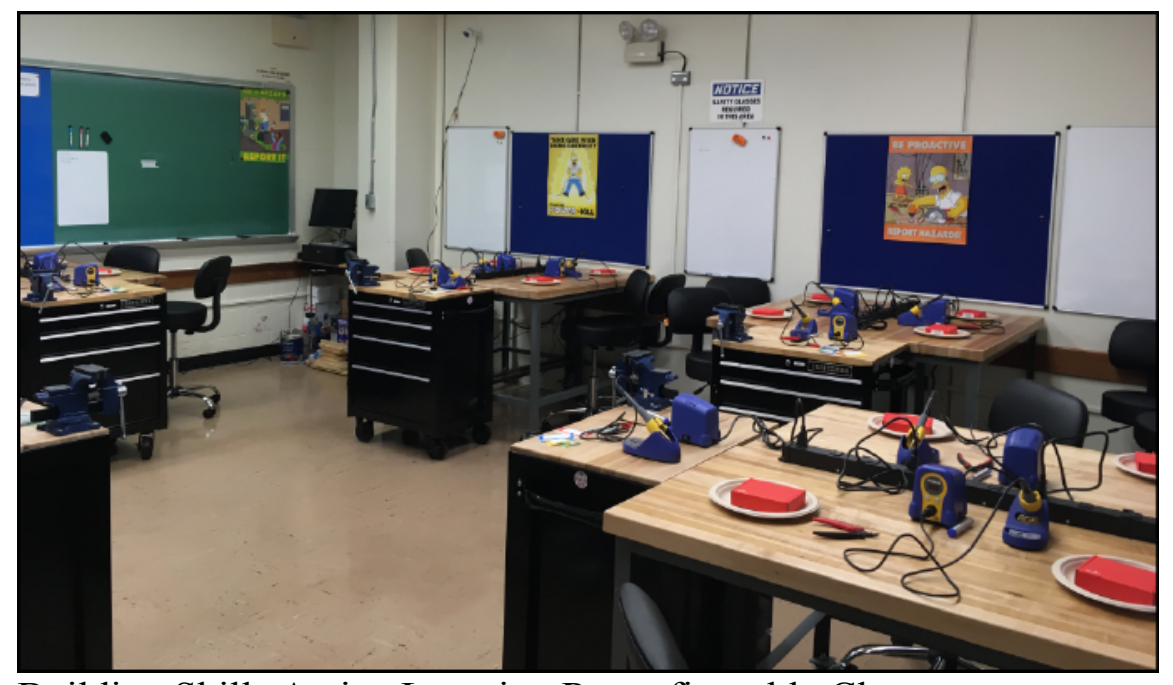

Building Skills Active Learning Reconfigurable Classroom

The first week of build group began with general safety procedures for the room (safety glasses required, long hair tied back, denim aprons provided for clothing protection, closed-toed shoes required, etc.) Each student was given an engineering laboratory notebook to use with a brief tutorial about good practice for engineering documentation in building.

Equipment in the rolling tool chests that were used during the builds included: hammers, various pliers, multiple types of screw drivers, ratcheting socket wrenches, adjustable wrench, a level, hex wrenches, measuring tape, calipers, hand saw, miter box, clamping vise, sanding sponges, cordless power drills, drill bits, corded and cordless Dremel rotary tools, various Dremel tool accessories and tips, wire strippers, soldering iron station, soldering helping-hand magnifying stand, tap and die set, and various types of small common hardware items (nuts, bolts, screws, nails, wire, shrink tubing, solder, staples, etc.). Items were selected for the tool boxes to balance beginning level concepts, specific project use, affordability, ease of use by students of all sizes, and common applicability to future self-building projects. Students were provided web links to all the tool specifics through the course management system page in case of interest in future individual purchase. 


\section{Projects \& Skills}

The projects in the build groups were designed to introduce uses of basic hand and power tools, increase confidence, expand making skills, and have students take home their individually made creations. The projects and skills in the build group were discussed informally each week by the faculty member in context of working in laboratories, internships, or student organizations across a variety of engineering majors, but also through personal building experience stories from the faculty member related to basic household maintenance skills.

Students sat in groups of 4 each week, and they discussed \& collaborated together, but when it came time to build students each individually used the tools to complete projects and were able to each keep projects they made during the build sessions. Student seating in groups of 4 was primarily used based of the combination of space and workbenches available in the maker-space classroom. Each weekly meeting was a 2-hour block of time in the makerspace classroom, which either involved one large project, or two mid-sized projects to take up the time. Projects were structured to last approximately 90 minutes, so if some students took a little longer they did not run out of time to complete their projects. If a student finished early, they were given the option of leaving early each week, or completing a second version of the project from that week or one of the earlier weeks if they had a hands-on item they wanted to create again.

Projects (and related tools included): wooden coasters (measuring, Dremel tools), string art (pattern planning, pliers, hammers, nails), wooden swiss-cheese (power drill, drill bits, vise), ratcheting wrench mini-practice (ratcheting wrench, hex keys, adjustable wrench), lighting up the LED (wire stripping, soldering iron, shrink tubing), Simon-Says learn to solder kits (soldering iron, solder, helping hands), threading a hole (tap and die set, nuts and bolts), building picture frames (measuring tapes, miter boxes, hand saws, sanding blocks, screwdrivers, framing staplers, wood filler, paint), and power drill medley (practice different settings on drill with various hardware to know when to use which settings).

\section{What prior level of hands-on building experience do you have?}

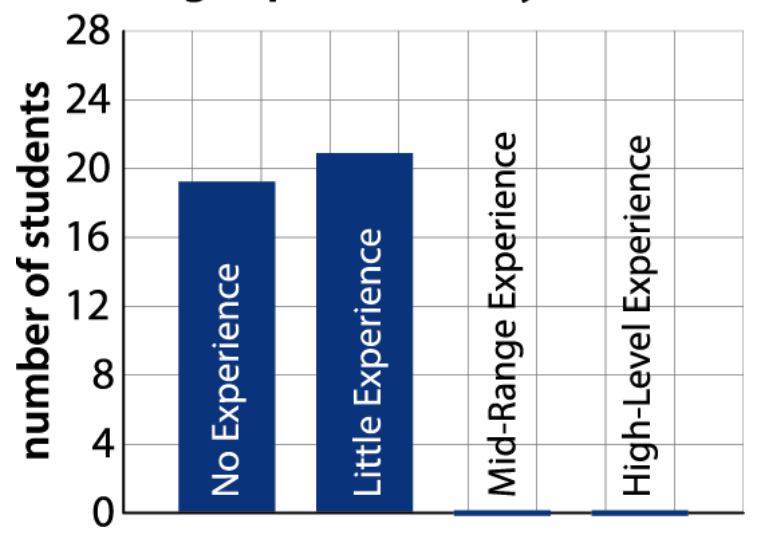

Self-reported student previous build experience.

Prior to participation in the build group, students were asked an open ended question about a skill they hoped to learn, some typical student responses are included here. 
If you could learn one new technical skill from this group what would it be?

"Soldering. I've never soldered, and quite a lot of simple things that I can otherwise assemble require it."

"I want to have a solid foundation on how to use power tools."

"I would love to learn how to use a hand saw."

"I would love to learn how to use a Dremel, I got one as a present but have no idea where to get started with it."

"I just want to learn how to handle tools properly and the safety that goes along with them."

"How to remove a screw with a manual screwdriver without stripping the screw."

"Being able to do more fixing around the house."

"I want to learn to use power tools. I want to stop being afraid of them. And I definitely want to improve my soldering skills."

\section{Art and Engineering Skills}

The first two projects students completed were creating wooden coasters and string art creations while learning to use tools. Both of these projects used wooden log slices as a base for the projects, and students were given instruction on the use of tools for each project, some practice time on scrap materials, and shown samples of the projects created by the faculty member and undergraduate peer mentor. In the wooden coaster project, the wood slices were pre-stained by the peer mentor, and various stencils were provided to students who might want patterns to trace, or they were welcome to create their own free-form sketches. They were shown the use of two different types of high speed Dremel rotary tools and various engraving tips for the tools. Students then were set to practice with the engraving bits on scrap wood, before choosing their wooden coaster and design to sketch and then engrave away with the rotary tools.

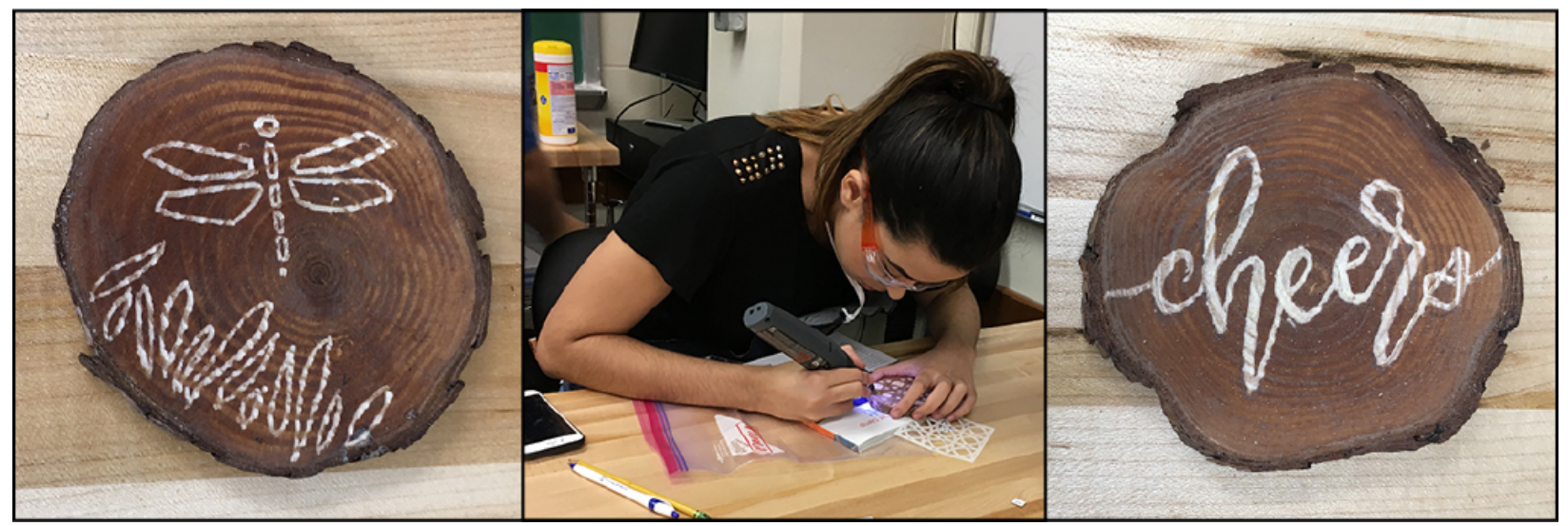

Samples of student created coasters and a student utilizing the Dremel rotary tool in the build. 
The send project was creating string art. Using the same wooden tree slices, students were given ideas of pre-made string art patterns or were welcome to design their own patterns on graph paper. They practiced carefully controlling hammer use, and using needle nose pliers with small finishing nails. The faculty member discussed engineering applications of small controlled hammers and nails relating to fitting prosthetic limbs, to tie into some of the items they might see use of simple hand tools in internships or other activities. The faculty member promoted a balance of allowing creativity through use of tools in artistic projects as well as the discussion of engineering applications of tools for a larger perspective of student use.

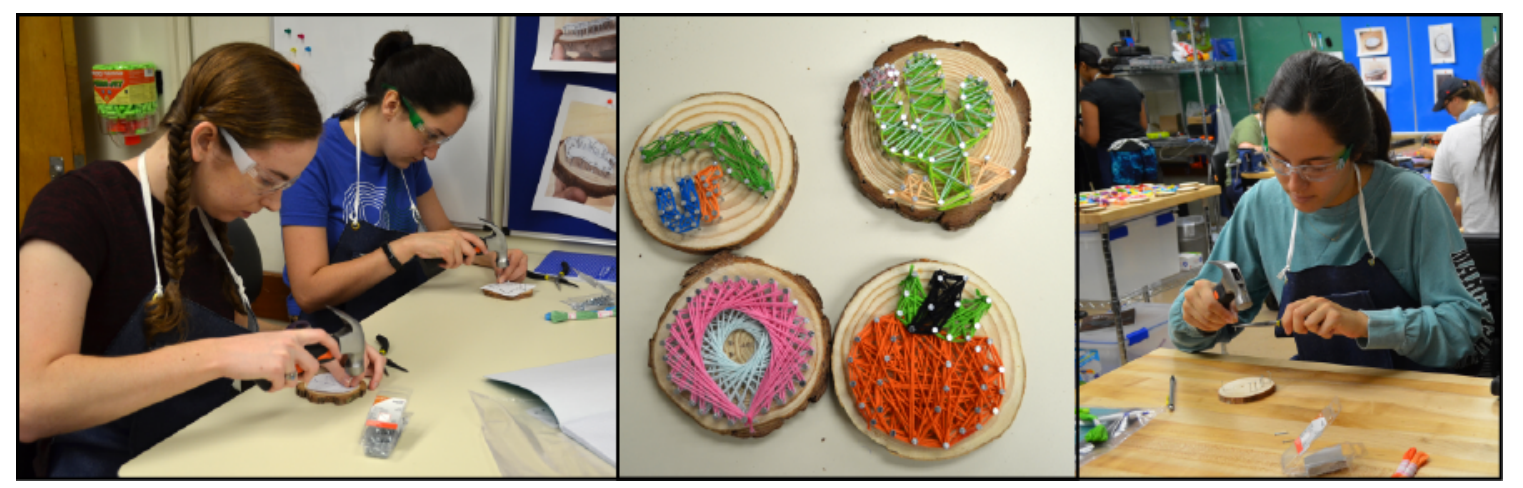

Students using hammers and needle nose pliers for string art with samples of student work.

The first two projects were ways to learn common hand and power tools through an artistic based project. Based on that structure, students were asked a survey question on the relationship between creating art with tools and tool mastery.
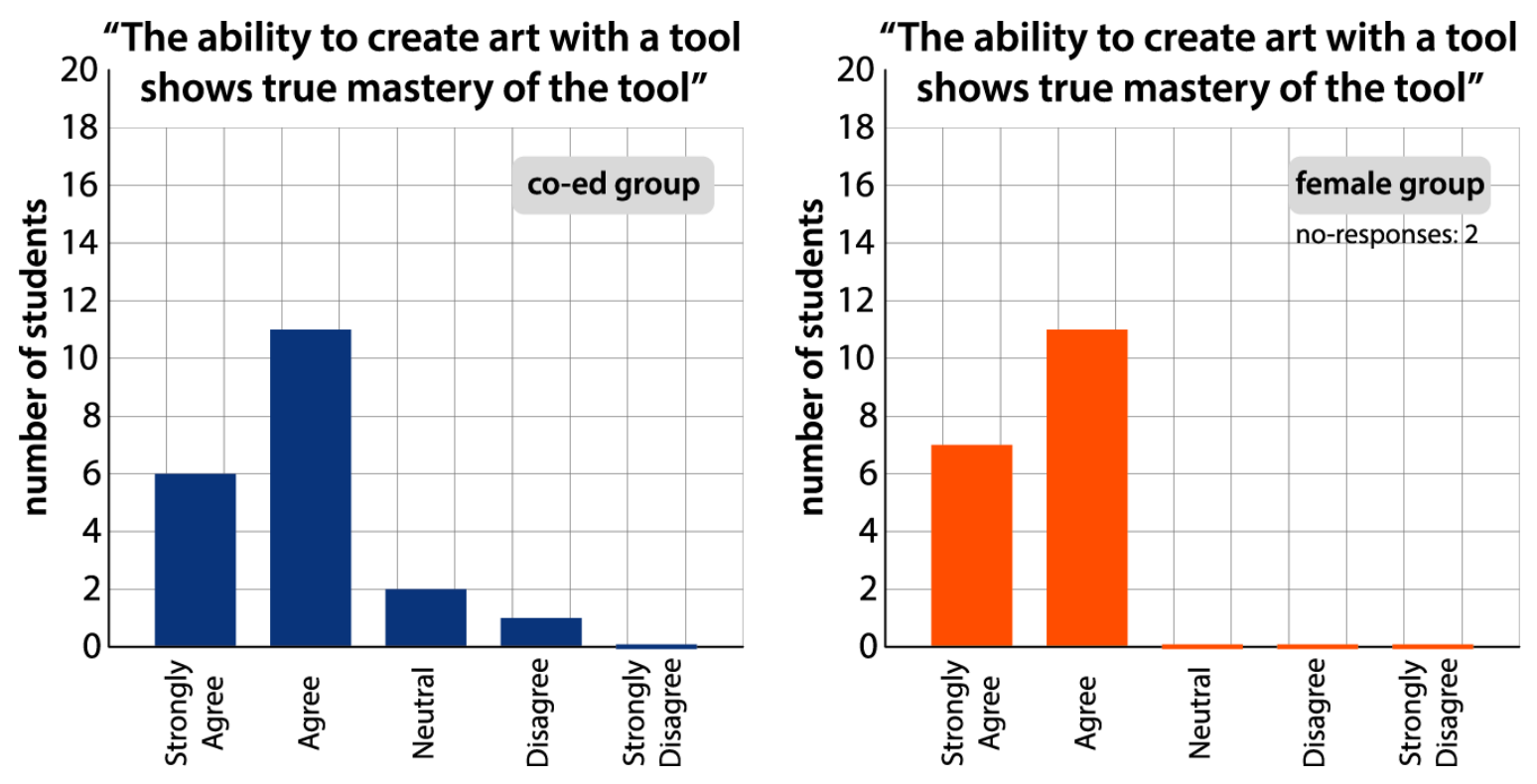

Following the second build, students were also surveyed on the build group environment. The goal of the group was to be a welcoming and inclusive place, where students felt no pressure of grades as well as no peer pressure while learning new skills. 

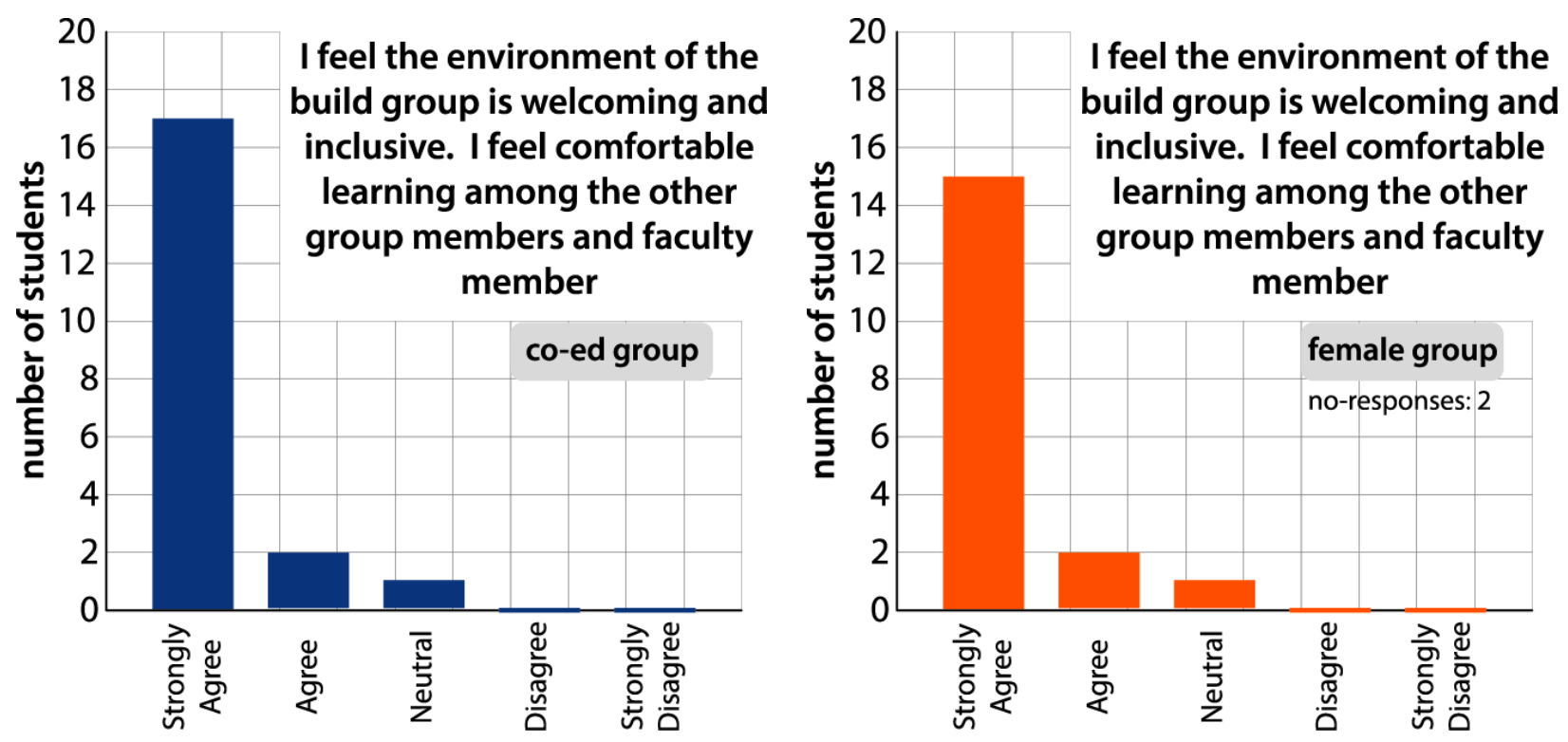

\section{Soldering}

One of the projects including learning to solder kits. Soldering is a skill many students expressed interest in learning, and there is no formal educational model on it within the for-credit engineering curriculum.
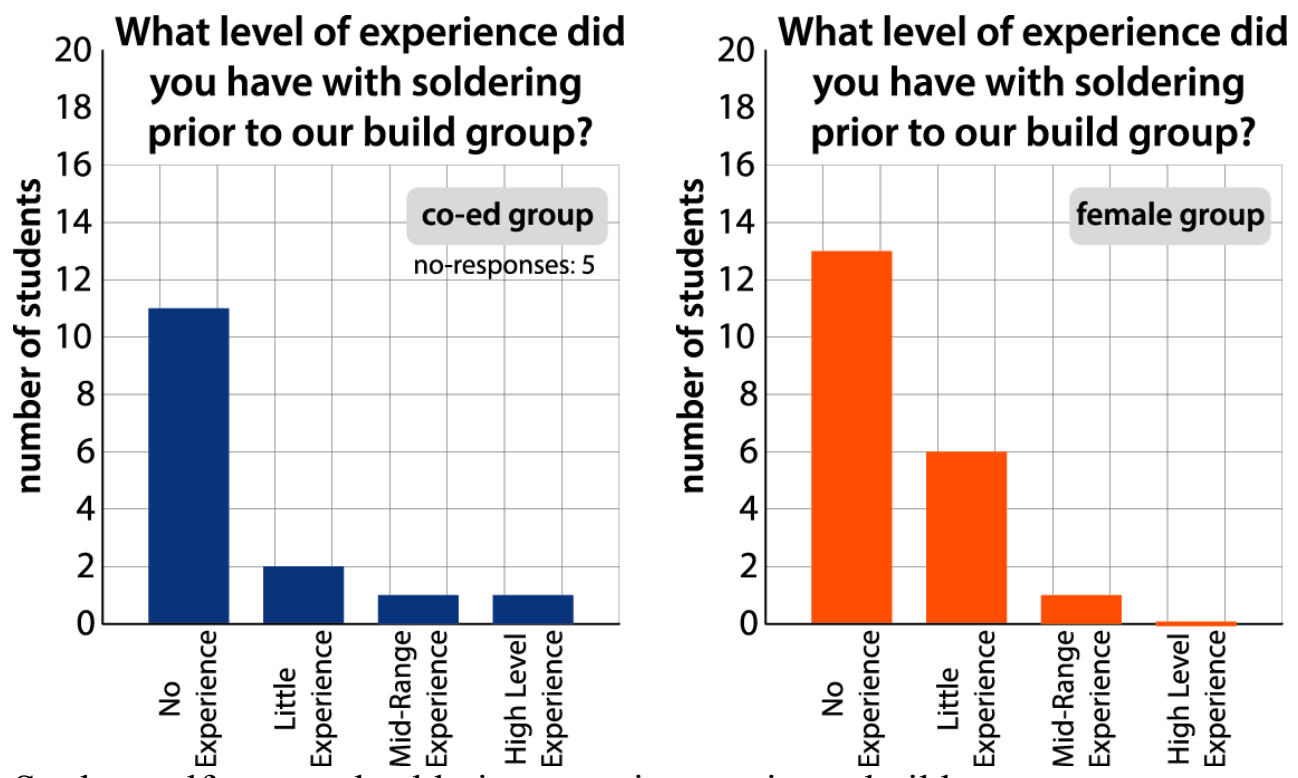

Student self-reported soldering experience prior to build groups.

For the soldering project, commercially available Simon Says Learn to Solder Kits from the SparkFun company were utilized. These kits used thru-hole soldering and were a good introduction to soldering for students new to the practice. In preparation, the faulty member performed all the steps of the learn to solder kits under an overhead camera and made a detailed video on the process, highlighting challenges and expectations of creating the kit for the students ahead of time. The students were tasked with watching the videos ahead of the build session to 
be well prepared for creating the kits and students also had the well structured paper instructions that came with the SparkFun Simon Says learn to solder kits in the group build session.

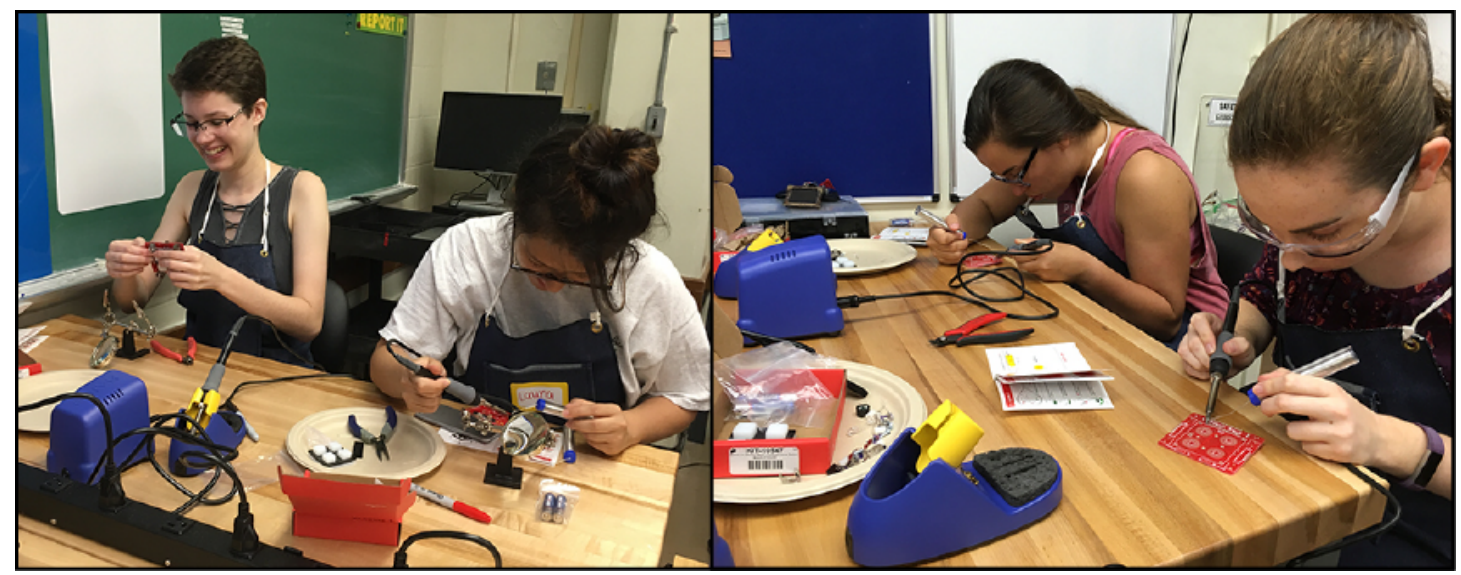

Students learning soldering by creating the SparkFun Simon Says Learn to Solder Kits.

One measure of success of the soldering exercise was the likelihood of students to solder independently in the future if they were provided access to the tools and workspace.
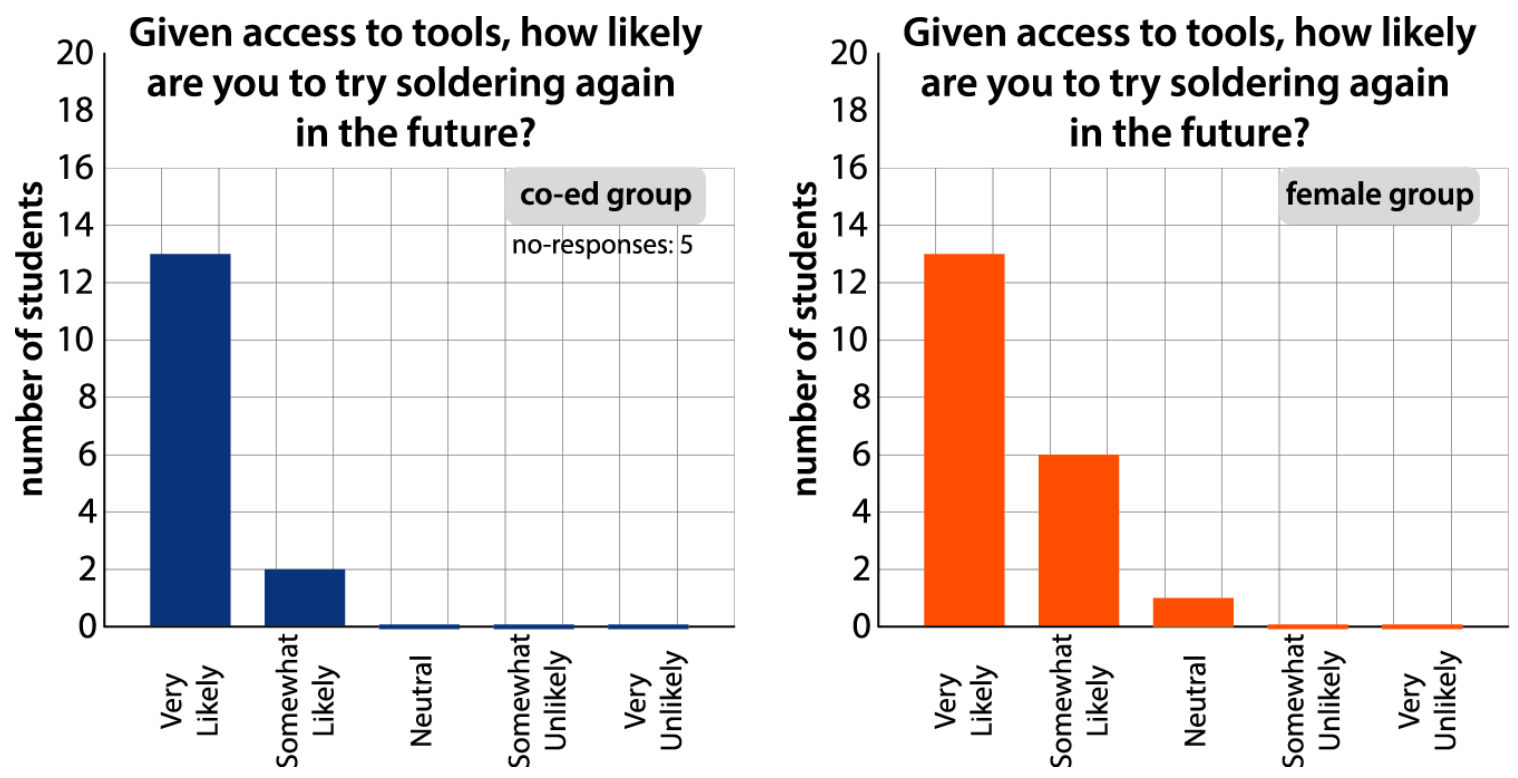

\section{Post-participation Goals Survey}

The goals in the formation of the Building Women in Engineering and Building Skills in Engineering groups were: a) Creating a safe environment for engineering students to learn, build, fail \& redesign, without pressure of grades or deliverables; b) Increasing individual student tool knowledge, basic making skills, and confidence, so when in group projects later they do not shy away from the design and building portion of team based engineering projects; c) Improving student skills for increased participation in engineering societies, internships, hands-on outreach projects, and student build teams; and d) Promoting a culture of making within all engineering students. Students were surveyed following their participation on their insight on these four 
specific goals. While goals c) and d) are broader impacts, for this pilot study students were surveyed on their self-reported perception of the impact on their skills and culture of making.
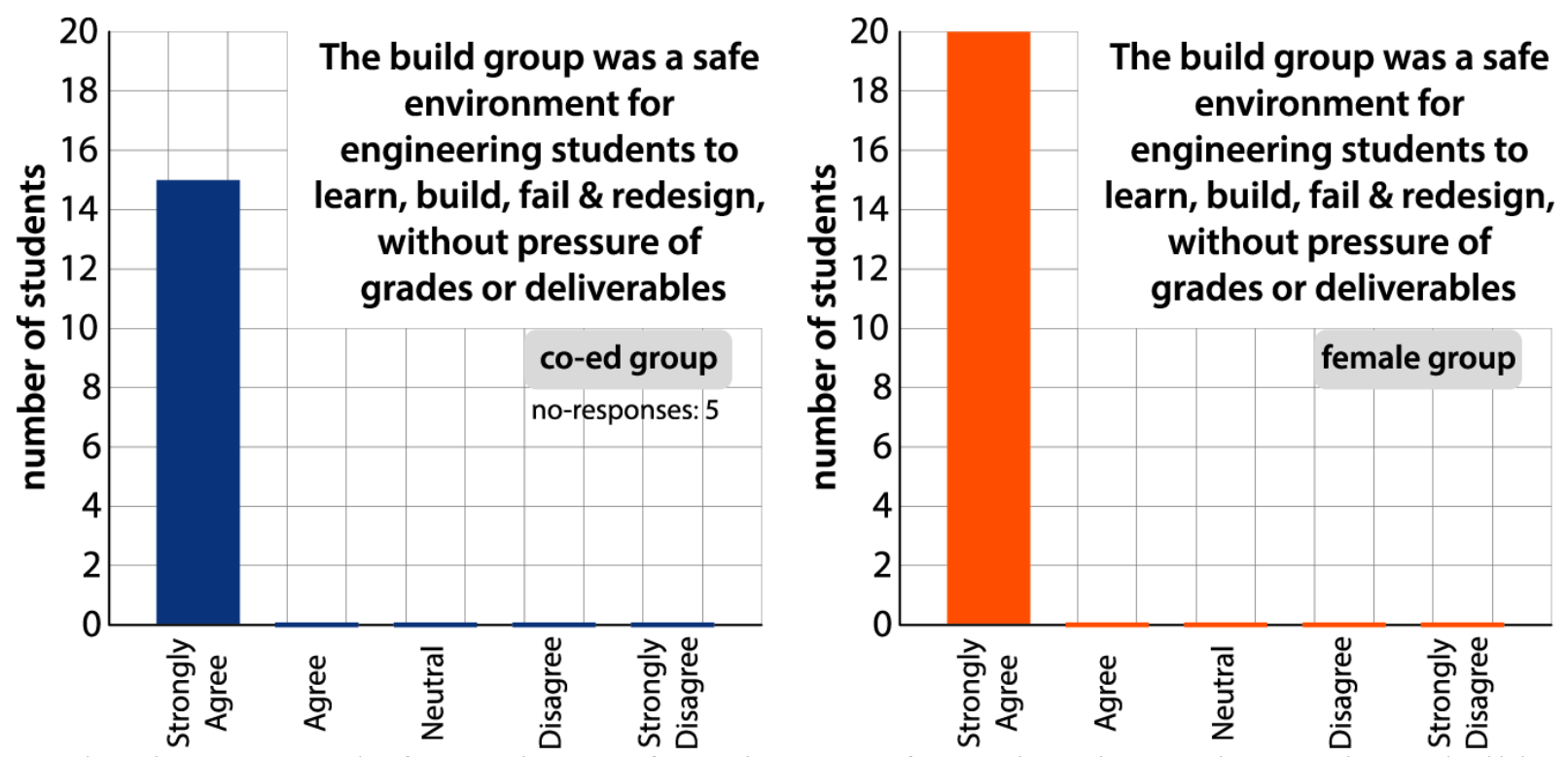

Student input on goal of: Creating a safe environment for engineering students to learn, build, fail \& redesign, without pressure of grades or deliverables.
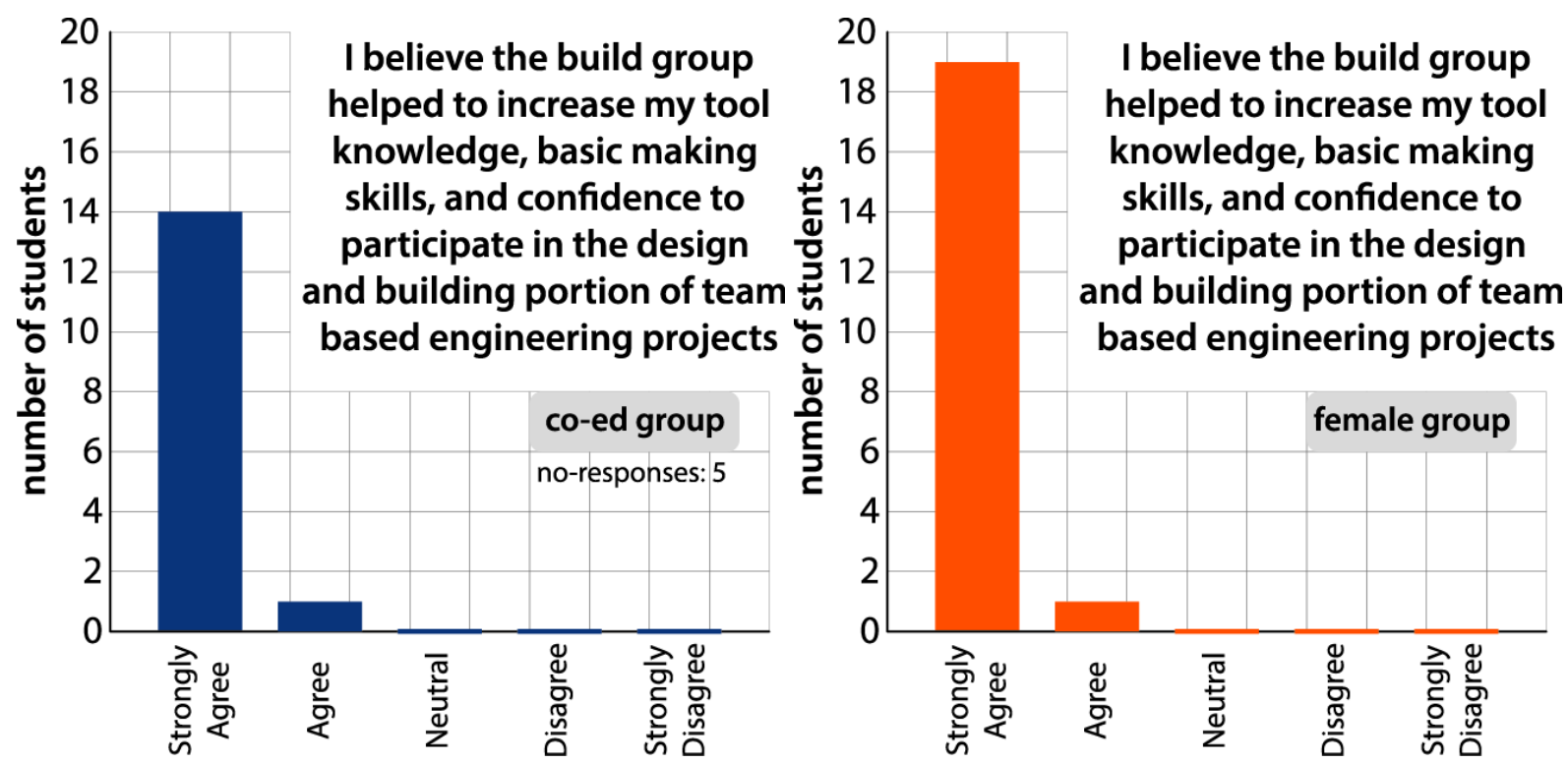

Student input on goal of: Increasing individual student tool knowledge, basic making skills, and confidence, so when in group projects later they do not shy away from the design and building portion of team based engineering projects. 

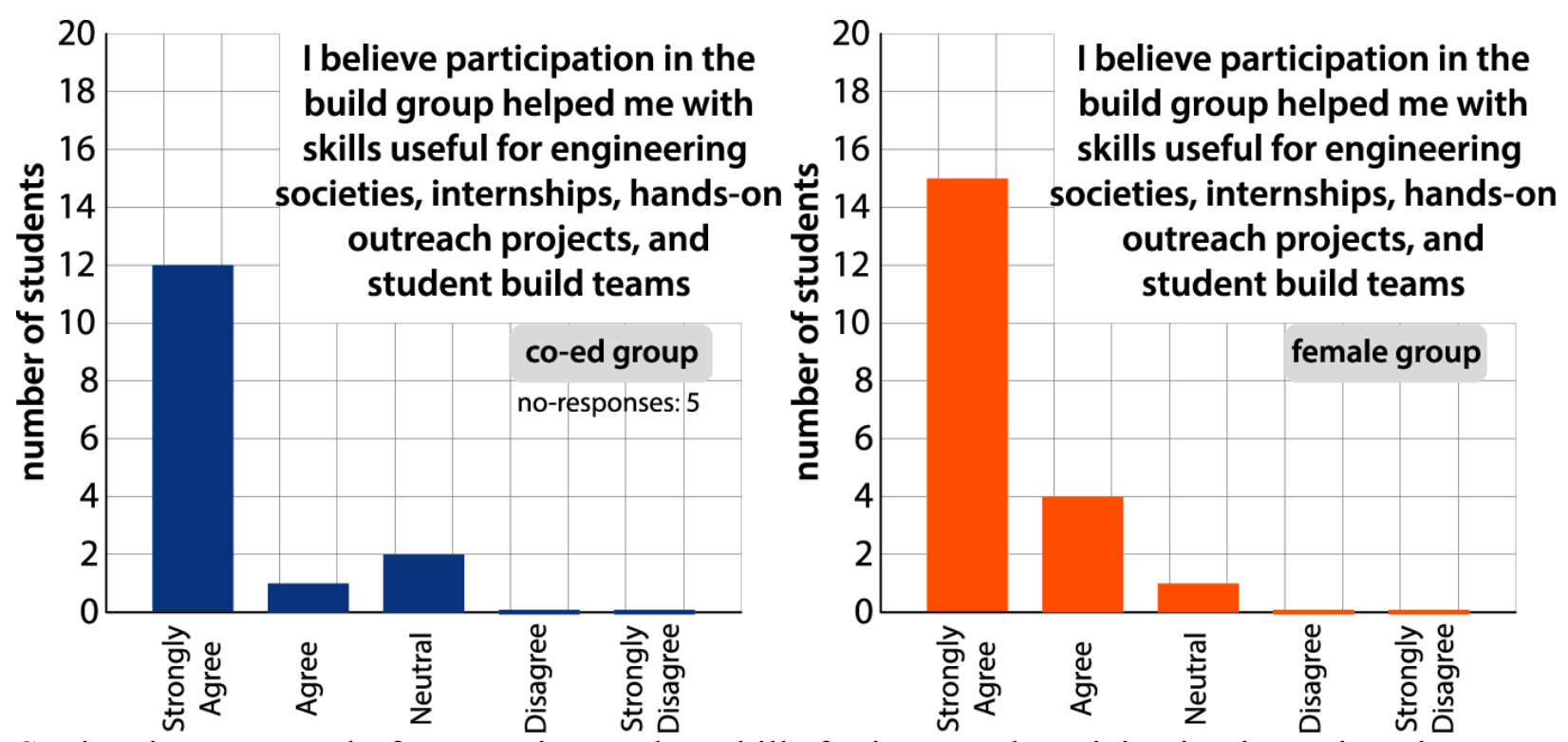

Student input on goal of: Improving student skills for increased participation in engineering societies, internships, hands-on outreach projects, and student build teams
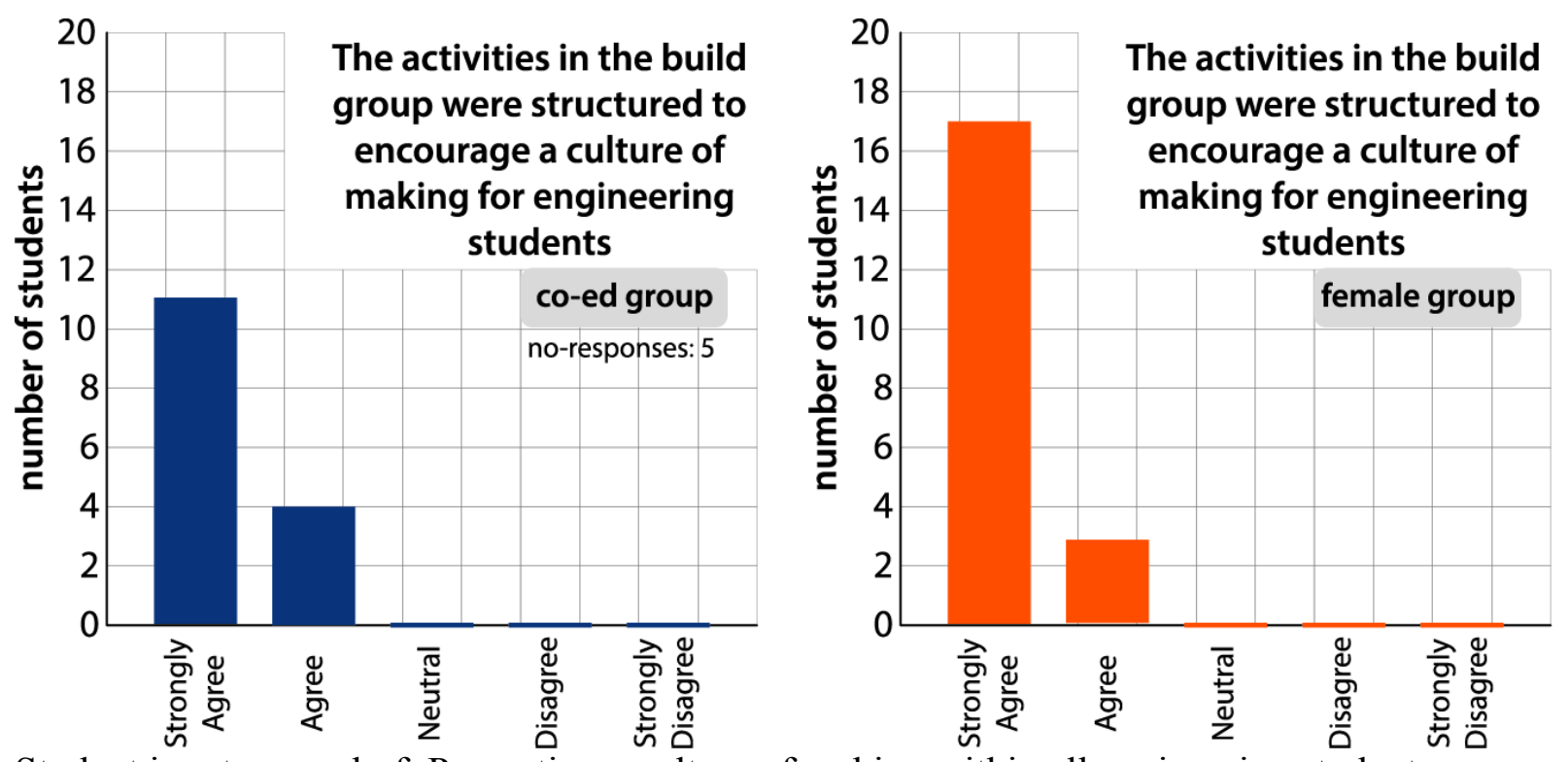

Student input on goal of: Promoting a culture of making within all engineering students.

\section{Impact}

Students were asked an open ended question at the end of the build group of What aspect of the building group had the most impact on you, and why? The student written responses to this question give the most realistic summary of the immediate and various aspects impact of this short seven-week hands-on building experience had on undergraduate engineering students. There are a number of quotes included below, separated by female and co-ed sections. While they may take up a number of paragraphs, the variety of ways students were impacted are important to the scope of this work and full student quotes were included. 


\section{Female Group Responses}

\section{What aspect of the building group had the most impact on you, and why?}

"The stress free environment biggest impact because I felt that I could relax about grades and really just enjoy the process"

"Working with a group of women who all had different levels of experience with each build from week to week was the most impacting for me. Every week, we would all combine our individual knowledge and experience to work together and complete the build as a team, which was the most rewarding to me."

"The aspect of flexibility made the biggest impact on me because it allowed the group members to realize how we can do anything that we set our mind to. If we wanted to do a side project, and the resources were there, we would just go for it."

"Seeing successful women in engineering. From the third and fourth year engineering students, to Dr. Dickrell herself, everyone in the group was someone I felt comfortable asking for help, but more than that, they were all people I want to emulate. I am so glad I learned many skills I will be able to use in various applications. But the most valuable thing I took away was hearing about a woman who had kids and a dog and managed a house and yet still had a successful career. That helped relieve some of the pressure I was feeling to make sacrifices, that I now know would be unnecessary."

"For me, the thing that keeps me motivated to pursue a STEM degree as a woman has always been community. Without the encouragement of other female engineers, it would be easy to give up or think yourself not good enough. This build group created an incredible bonding experience for me and my fellow female engineering students. I loved learning around the low-pressure environment created by a room full of girls all learning new skills together, especially with an instructor who is a successful female engineer."

"The aspect of build club that has the most impact on me would be the amount of confidence I have gained. I am no longer afraid to use tools and I no longer think that I need help from my male friends to do something simple like hang a picture frame or use a drill. I loved the inclusive environment and the constant support that I experienced and I would wholeheartedly recommend this club to all my peers."

"Dr. Dickrell kept saying, "it's okay if you don't get it right the first time" or "don't worry if you make a mistake, you can always come back to do the project again" which took the pressure of having to do everything right away. It was huge for me because I didn't want to disappoint her or the people making this build group happen with all the supplies and their times. Even if I made mistakes, I only heard encouraging words from Dr. Dickrell, it really made a positive impact on my learning experience."

"The low stress, community environment put forth in the build group was my favorite aspect because it allowed me to take on new projects without constantly stressing about messing it up and holding someone back because I didn't understand something. By treating every project as if 
we had no experience (which was usually true for me) I was able to keep up with everyone else in the group and ask for help if I didn't understand a step."

"The most impactful part of the build group to me was that it provided me with hands-on, technical experience that I had never been exposed to before. Getting that experience as a freshman in college was very helpful, as I am going into a career in engineering, and the skills in this build group are crucial to that career. It was also impactful because it fostered personal growth and confidence-building. Getting to know and use some powerful tools, surrounded by women in engineering who are doing the same, was very empowering."

\section{Co-Ed Group Responses What aspect of the building group had the most impact on you, and why?}

"Access to tools, I've never had access to before. I was raised by a single parent and whatever she had and could use was what I was taught to use, but not much more. We repaired and installed some things but that was it. Other tools that wouldn't be reliably used were often too expensive for too little gain. Having access to those tools now, without having to outright buy them, and then access to someone to give guidance is invaluable. This class has meant much more to me than just the sticker price of the tools. I hope to use the skills I learned in both my career and my free time. I really hope the build club can be expanded so I can recommend it to more people."

"Easily the process of design and manufacturing. The build group gave an opportunity to see a project go from initial diagrams to finished product. It gave me a greater sense of confidence for future projects."

"I liked being able to work with the people around me to figure out how the tools worked and how to properly use them. Sure, I could've asked for further instruction, but I feel like it made me more comfortable working with others by asking those around me and working together with them to figure things out."

"I loved learning how to use power tools. When I was younger I was told they are dangerous, so I was afraid of them. As I got older I realized that even if they are dangerous they are very useful and can be handled safely with proper training. This Build group was my first opportunity to receive proper training with power tools. Thanks to this training, I am comfortable using power tools on my own now. learning to confidently use "dangerous" power tools, is empowering."

"The build group made engineering fun for me again. The constant stress of exams and graded assignments feel as though they are beginning to take a toll on me. The build group created a stress free environment where I felt that I could fail countless times and it would be OK. With a positive attitude I can keep on going and eventually succeed and learn what I want to do.

Engineering is about experimentation and creating new products, ideas, and technology. But with that comes failure. I feel that school conditions students to become afraid of failure and to stick to what their comfort zones are and follow a path that is guaranteed to work. But engineering needs risk taking to continue to innovate. In short, the build group made me more comfortable with messing up and starting again." 
"The aspect of the Build Club that had the most impact on me was the ability to engage with new people that I would likely have never met on my own. I liked being able to come together to learn new hands-on experiences with people who shared the same classes I did and feeling like I was more part of the engineering community on campus."

\section{Gender Environment Feedback}

The students both groups were given an open ended survey question about the impact of having either a female-only section or a co-ed section, while lengthy, the student responses are included below in their full content as they speak best through their voices on their thoughts on gender and the building environment.

\section{Female Group Responses}

Please tell me how having a female-only build group impacted your participation? Did it make a difference? If you believe it was important, why?

"I think it was important because more often than not we are the minority in classes and groups within classes, so it was refreshing to be surrounded by and work only with females. I would also benefit in the same aspects that I did if I was in a co-ed group though."

"In the upper level engineering classes, it is starting to become more male dominated. By having this club, it is a good change of pace and more comfortable setting to ask questions." "I felt like there was nothing to prove. No embarrassment. No feeling like you needed to hold back and let someone else do it. But I think that was due to the "get your hands on it" attitude of the club more than the gender."

"I feel like the female only environment just made for a really interesting environment because most of the time females are outnumbered in most engineering disciplines and having a room full of girls who face similar issues is nice."

"I liked being in the female only build group even though it felt a little exclusive. I liked some of the topics that were brought up that were just life related and not specifically career related."

"Though a co-ed group would have still been enjoyable, I enjoyed the comfort of learning alongside other women who know the feeling of being 'man-splained' or put down for being a woman in STEM. There was much less pressure placed on you. Also, it was fun to see fellow SWE members around! It was a nice bonding experience with fellow female engineers we are likely to run into elsewhere!"

"I loved having a female only build group because we all became comfortable being around each other and everyone was so quick to help. I am not sure how comfortable I would be if there were men around, since they tend to assume they know more and want to show off (this is a general statement and doesn't speak to all men, just the experiences I have had). Being a woman in STEM is hard enough as it is and its nice to not have the constant competition from our male peers." 
"A female-only build group didn't greatly change my participation level, but it did ease some nerves inside. With men there's always a level of competiveness, in my opinion, to prove that you can do the same thing just as well. In a learning environment, however, I prefer to work without that level of internal stress. It's also nice to work with other females because I appreciate the level of detail that is generally put towards crafting. I believe it's all about personal preference."

"Truthfully, I only chose the female-only group because it worked better in my schedule but I did feel more comfortable learning with other girls. It made me feel like I wasn't the only girl in my classes who didn't know what she was doing."

"Having a female-only build group made me feel more comfortable when completing these projects. Usually in my engineering courses, I feel that men have more knowledge in certain topics and I most likely have not worked with it before. Therefore, I liked the idea that I was working only with females and was not worried about having no prior experience."

"I feel like it was more comfortable because there was no issue of what is known as "mansplaining" so I didn't feel inferior to anyone there, but only equal. Also guys tend to be louder and messy so it was nice not to have to deal with that."

"I think it was a safe environment for the beginners. It was free of judgement from guys. It gave us the extra push we need since we have been discouraged to jump in on fixer-upper situations. I am so grateful for people like Dr. Dickrell who are looking out for young women in STEM fields. She made it such a fun experience for us all and is making a huge difference in our careers and self confidence."

"Sometimes it feels like the male engineers have more experience in using tools like the ones we used in the build group than female engineers. Also, engineering is still a field that is maledominated, and it's refreshing to be in an engineering-esque environment in which the minority are the only ones there. I'm not sure if it would have decreased my participation by having men in the room too, but I feel as though the female-only environment was preferable for the reasons I've already mentioned. I think it is important that there is a place for women to go and learn together apart from the male majority to make them more comfortable and to create friendships to help each other navigate the male-dominated industry."

"It was a more comfortable environment for me, as we all had similar backgrounds with the tools. I did not feel ashamed to say I had no experience with some power tool. Not to stereotype an entire group of people, but oftentimes in class or clubs, I hear guys talk about concepts with ease, and they are absolutely shocked that I struggle with that concept or that I have never built my own house out of Legos or built a car with my dad or some random male-bonding activity. Learning is a lot more fun when you don't feel behind all of the time, and it felt good to be around people who haven't been exposed to building/designing/engineering all of their lives."

"I wanted to be a part of a female-only build because they would be more likely to understand the feeling of always being behind our peers in terms of skills because of what was previously 
offered to us. On top of this, we were able to consult each other about stigmas that we faced within our major because of our gender, and how to move past those stigmas."

\section{Co-Ed Group Responses \\ Please tell me how having a mix of genders in the build group impacted your participation? Did it make a difference? Did you feel uncomfortable in any way, or were participants generally friendly regardless of gender?}

"Having a mix of genders allowed me to present my gender with ambiguity, as I do everywhere right now. Being transgender, I am very early in my transition so it's important that I feel at least moderately comfortable regarding that if I'm going to be learning. Sometimes people express negative opinions about who I am and that can be a very high stress situation (with occasional concerns for safety). Both women and men were all very welcoming and I appreciated it. I had very little stress about my perception and treatment by other people. It also felt okay to make mistakes and be in a sort of "practicing" stage, maybe because that's the point of the course."

"I didn't get the usual "I am male so I'm a better engineer than you" impression from this group. I don't think it made a difference but I'm glad there were more females present."

"I thought it went well. I don't think it made a difference in my performance. It was a nice mixture of individuals."

"I actually made some friends I would not have run into in my everyday life because we have different interest, but I was very intrigued to hear their stories and I really enjoyed the spirit of the group overall."

"I don't think it made a difference, everyone was friendly and easy to get along with, plus I feel like it didn't really matter for the gender because we were just focused on our task and the methods to go about what we had to do."

"Having a mix of genders did not impact my participation, and I did not feel uncomfortable. If anything, I felt relieved that my gender was not the only one who felt the need to take a class on building skills."

"I've always been comfortable around men, so the mixed genders didn't bother me. I would've felt awkward if everyone else was at a much higher skill/tool knowledge than me, but it seemed like most were at the same level so the environment felt very supportive."

"Having mixed genders didn't make me feel uncomfortable at all, I didn't mind that aspect of the experience."

"Having a mix of genders in the build group did not have any impact on my participation since most, or all, of my classes, have a mix of gender, and male students are in a higher ratio than 
female students. I have adapted to learn how to work/communicate with male engineering students through my classes' projects."

"The mix of genders did not impact my experience at all. I don't usually pay attention to things like that. Everyone was very friendly and had a great attitude."

"I felt comfortable with a mix of genders. It was a comfortable environment where everyone got along. Everyone was very nice and respectful."

"The mix of genders didn't impact my participation in the build group at all. I was comfortable with all participants as everyone was so friendly, and was able to make a few friends from the experience!"

"It made me feel good that the boys were at the same level as me. I wasn't uncomfortable at all they were all very nice. In general, with the whole group no one looked down on another. They were all very encouraging."

"Participants were friendly regardless of gender. Felt comfortable in the build club environment."

"I don't think it made a difference other than breaking the stereotype that guys know everything about tools. I did not feel uncomfortable in any way. Everyone seemed friendly."

\section{Future Work}

The Building Women in Engineering and Build Skills in Engineering groups are due to be continued in their current state, rotating new hands-on projects in to optimize different use of the tool carts developed. In the semester of publication, the next round of registration opened for build groups with 135 applicants for the limited 40 seats of the two build sessions. While difficult to turn down 95 students due to space and time resources, it is rewarding to know so many students are interested and will take advantage of this ongoing resource. The next step will be to investigate external sources to ensure continued funding of current students participating and potentially expand the number of student sections each semester to accommodate demand.

Advanced Building in Engineering. The semester following the submission of this work, a new Advanced Building in Engineering group was launched open to previous participants in the introductory build group. The advanced group will take on small group projects, learning skills of solid modeling, 3D printing, Arduino hardware/software to make a team design project. The first project will be for students to design and construct a self-watering windowsill planter using Arduino sensors/actuators for control/motion, and solid-modeling/3D printing for creating parts. 